\title{
Electromagnetic Hypersensitivity- A Disease of the New Era?
}

\author{
Jawad Ahmed ${ }^{1 *}$, Farheen Malik ${ }^{1}$ and Junaid Ahmad ${ }^{2}$ \\ ${ }^{1}$ Department of Internal Medicine, Dow University of Health Sciences, Karachi, Pakistan \\ ${ }^{2}$ Department of Internal Medicine, Liaquat University of Medical and Health Sciences, Jamshoro, Pakistan
}

\section{Respected Editor,}

Electromagnetic hypersensitivity (EHS) is a cluster of nonspecific symptoms in which the affected person believes are due to exposure to electromagnetic field (EMF). EMF is generated by power lines, Wi-Fi routers, wireless devices and mobile towers. Individuals most commonly complain of redness, tingling, and burning sensation in the skin, and somatic symptoms such as fatigue and tiredness, problems in concentration, nausea, heart palpitation, and digestive disturbances which affect their daily life. In 2005 WHO proposed the name "Idiopathic Environmental Intolerance Attributed to EMF" (IEI-EMF) for EHS symptoms [1].

The prevalence of IEI-EMF varies globally, being $4.6 \%$ in Taiwan [2] and 7\% in Germany [3]. However, no such study has been conducted in Pakistan to find out its prevalence. The study published by Huang PC et al. in January 2018 showed that women had a higher likelihood of reporting symptoms of IEI-EMF compared to men [2]. People with IEI-EMF reported physiological symptoms such as numbness, tingling, burning skin, memory loss, and concentration difficulties and poor health-related quality of life [4]. On the contrary, there are studies that failed to establish a significant relationship between the onset of symptoms and electromagnetic field exposure of an individual $[5,6]$.

A study by Tseng MC et al. showed that the risk of self-reported electromagnetic field sensitivity (SREMFS) was higher among individuals who reported low health status, who were incapable of working, and who had coexisting psychiatric illnesses. Almost one-third $(30.7 \%)$ of individuals with SREMFS had psychiatric comorbidity [7]. A study based in Taiwan found a positive association between IEI-EMF prevalence among individuals and coverage of issues related to EMF by media. As media coverage about EMF related issues decreased from 2005 to 2012, the frequency of IEI-EMF also decreased [8].

However, the effects of EMF on different tissues and animal models have been studied in great detail since

*Corresponding Author: Jawad Ahmed, Dow Medical College, Dow University of Health Sciences, Karachi, Pakistan.

Email: jawadahmedd13@gamil.com

Received: January 01, 2020; Revised: February 02, 2020; Accepted: February 06, 2020 DOI: https://doi.org/10.37184/lnjpc.2707-3521.1.12 the early 1970s. In 1973, Tolgskaya and Gordon published their work titled as "Pathological Effects of Radio Waves" based on experiments on different animals and tissue models. They reported that tissues of the nervous system were most sensitive to radio wave (non-thermal EMF) exposure followed by cardiac and testicular tissues. They also noted that pulsed electric fields more actively produced histological changes in tissues than non-pulsed fields. Non-thermal EMFs were also found to adversely affect the secretions from neuroendocrine glands especially the hypothalamus and the pituitary, initially increasing the secretions followed by a decline or "exhausted" phase [9]. EMF produces its effect by activation of voltage-gated calcium channels (VGCC). Pall ML published an extensive review of literature summarizing the effects of EMF. He concluded that higher sensitivity of CNS to EMF is due to higher numbers of VGCCs within neurons and neuroendocrine cells. Increased $\mathrm{Ca}+2$ release for prolonged duration damages the cell and can even lead to apoptosis [10]. The study published by Pilla AA also showed that EMF caused activation of VGCCs. He found that exposure of neuronal cells to pulsed microwave EMF caused an immediate peak in calciumcalmodulin-dependent nitric oxide release thus supporting the effects of EMF on VGCCs [11].

Despite the controversy on the subject of IEI- EMF, WHO Fact sheet $\mathrm{N}^{\circ} 296$ infers that physicians should focus on the clinical case of affected individuals rather than an individual's perception to reduce EMF exposure. Furthermore, evaluate the patient for any medical or psychiatric/psychological etiology that can give rise to symptoms and assess the patient's home and workplace for any causative factor such as indoor pollution or poor lighting. For long-lasting symptoms, therapy should be aimed to reduce them [1].

The physician should establish a good relationship with the patient and counsel them. Many articles written by individuals suffering from EHS are available online which suggest ways to reduce EMF exposure. The following are a few things mentioned in them:

1. Turning Wi-Fi off when not being used.

2. Keep mobile devices away from your body.

3. Try to minimize the use of microwave ovens.

4. Do not use Bluetooth headsets/earphones. 
In conclusion, if a patient complains about symptoms of EHS, the physician should inquire in detail about other medical conditions as well as the surroundings for sources of high EMF such as mobile network towers and treat the cause accordingly.

\section{CONFLICT OF INTEREST}

The authors declare no conflict of interest.

\section{ACKNOWLEDGEMENTS}

Jawad Ahmed and Farheen Malik contributed to the manuscript design. All authors significantly contributed towards manuscript preparation. Junaid Ahmad critically revised the manuscript. All authors have read and approved the final version of the manuscript.

\section{REFERENCES}

1. WHO. Fact Sheet No. 296: Electromagnetic fields and public health. World Health Organization; 2005. Available at: http://www.emfandhealth.com/WHO EMSensitivity.pdf (Assessed on: December 29, 2019).

2. Huang P-C, Cheng M-T, Guo H-R. Representative survey on idiopathic environmental intolerance attributed to electromagnetic fields in Taiwan and comparison with the international literature. Environ Health 2018; 17(1): 5.

3. Lauff $\mathrm{H}$, Wachenfeld A. Differentiated consideration of the use and perception of mobile radio - project FM8854. LINK Institute for Market and Social Research GmbH, Frankfurt am Main, 2014.
4. Kjellqvist A, Palmquist E, Nordin S. Psychological symptoms and health-related quality of life in idiopathic environmental intolerance attributed to electromagnetic fields. J Psychosom Res 2016; 84: 8-12.

5. Verrender A, Loughran SP, Anderson V, Hillert L, Rubin GJ, Oftedal $\mathrm{G}$, et al. IEI-EMF provocation case studies: a novel approach to testing sensitive individuals. Bioelectromagnetics 2018; 39(2): $132-43$.

6. Rubin GJ, Nieto-Hernandez R, Wessely S. Idiopathic environmental intolerance attributed to electromagnetic fields (formerly 'electromagnetic hypersensitivity'): an updated systematic review of provocation studies. Bioelectromagnetics 2010; 31(1): 1-11.

7. Tseng M-CM, Lin Y-P, Cheng T-J. Prevalence and psychiatric comorbidity of self-reported electromagnetic field sensitivity in Taiwan: a population-based study. J Formos Med Assoc 2011; 110(10): 634-41.

8. Huang P-C, Li K-H, Guo H-R. Association between media coverage and prevalence of idiopathic environmental intolerance attributed to electromagnetic field in Taiwan. Environ Res 2018; 161: $329-35$

9. Tolgskaya MS, Gordon ZV. Pathological effects of radio waves. $1^{\text {st }}$ ed. New York/London: Consultants Bureau 1973.

10. Pall ML. Microwave frequency electromagnetic fields (EMFs) produce widespread neuropsychiatric effects including depression. J Chem Neuroanat. 2016; 75: 43-51.

11. Pilla AA. Electromagnetic fields instantaneously modulate nitric oxide signaling in challenged biological systems. Biochem Biophys Res Commun. 2012; 426(3): 330-3. 